\title{
Variations in Lengthening of Bunches in Bunch-Train Operation in CESR *
}

\author{
$\underline{\text { Z. Greenwald }}^{\dagger}$, D. L. Rubin, Cornell University, Ithaca, NY
}

\begin{abstract}
In the Cornell Electron Storage Ring (CESR), which is operated with nine trains of five bunches, bunch lengthening results from the interaction of the bunch with the wake voltage induced at the hundred sliding joints along the ring. The decay time for the bunch induced voltage is longer than the time between bunches. Therefore the voltage witnessed by successive bunches will depend on both the resonance frequency of the sliding joint cavity and the arrival time of the bunches. In this paper we study the differences in the equilibrium bunch lengthening within the train and between trains due to the wakes induced in the sliding joints at two different resonance frequencies. One to achieve minimum power loss and the second to achieve minimum bunch lengthening.
\end{abstract}

\section{INTRODUCTION}

The longitudinal lengthening of bunches, as well as the heating in the sliding joints, depend on the wake field induced by the bunch train going through the sliding joints in CESR. The pattern of the wake induced in the sliding joints depends on the sliding joint's trapped mode resonance frequency, the decay time of this mode, and the spacing between the bunches. The sliding joint openings in CESR, which determine the frequency of the trapped mode, were optimized experimentally for minimum heating [1]. Unfortunately, the conditions to achieve minimum heating are different from those required for minimum bunch lengthening. In this article we study the spread of the bunch lengthening in the trains at these two conditions.

\section{WAKE INDUCED BY A BUNCH TRAIN}

The single bunch wake field in a sliding joint calculated using MAFIA has one dominant trapped mode [2] with resonance frequency, $f_{r}$, and a decay time, $\tau$. Since the decay time of the sliding joint is much larger than the spacing between the bunches, there will be an accumulation of induced field in the sliding joints due to passage of bunch trains. The field accumulated in each sliding joint will be different, causing a different temperature rise in each sliding joint and a spread in the lengthening of the bunches in the trains. The wake field of a single bunch in a sliding joint, $W_{b}(t)$, can be approximated:

$$
W_{b}(t)=A_{w} \cdot F w \cdot \epsilon^{\frac{-t}{\tau}} \cdot \sin \left(2 \pi f_{r} t\right)
$$

where $A w$ is the amplitude of the wake exited by $1 \mathrm{~cm}$ bunch, and $F w$ is a correction factor for the variation in the

\footnotetext{
* Work supported by the National Science Foundation.

† Email: zipi@cesr10.Ins.cornell.edu
}

bunch length (see Section 5). The wake, $W_{t}(t)$ induced in a sliding joint by bunch trains is:

$$
\begin{aligned}
& W_{t}(t)=A_{w} \cdot F w \cdot \sum_{r=0}^{N_{\text {turn }}} \sum_{n=0}^{N_{\text {train }}} \sum_{i=0}^{N_{b} \text { unch }} \\
& \quad \exp \frac{-\left(t-i \cdot t_{\text {bunch }}-n \cdot t_{\text {train }}-r \cdot t_{\text {turn }}\right)}{\tau} \\
& \sin \left(2 \pi \cdot f_{r} \cdot\left(t-i \cdot t_{\text {bunch }}-n \cdot t_{\text {train }}-r \cdot t_{\text {turn }}\right)\right) \\
& t_{\text {tunch }} \text { is the time between bunches. } \\
& t_{\text {train }} \text { is the time between trains. } \\
& t_{\text {turn }} \text { is the revolution time around the ring. }
\end{aligned}
$$

At the present there are nine trains circulating around the storage ring, $t_{\text {turn }}=2.56 \mu \mathrm{sec}$. The trains are separated by $t_{\text {train }}=0.22844 \mu \mathrm{sec}$. There are five bunches in each train spaced by $t_{\text {bunch }}=14 \mathrm{nsec}$. Figure 1 shows the detailed wake potential at the passage of the third bunch in the second train, while Figure 2 shows the wake accumulated during the second bunch train.

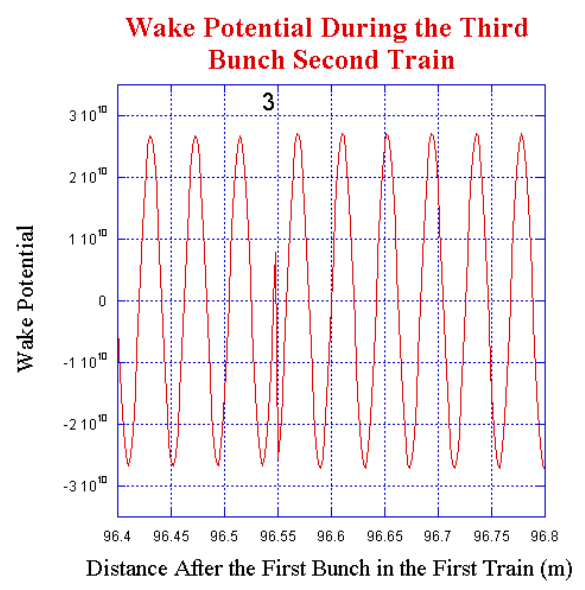

Figure 1: Induced wake potential during the passage of the third bunch in the second train. Note, the phase and amplitude change around $96.55 \mathrm{~m}$.

\section{THE CONDITIONS FOR MINIMUM HEATING IN THE SLIDING JOINTS}

The power dissipated by bunch train in the sliding joints is proportional to the integral on the wake potential from $-\infty$ to $\infty$ :

$$
P \propto \int_{-\infty}^{\infty} W_{t}(t) \cdot d t
$$

To get the minimum heating we find the derivative $\frac{\partial P}{\partial f_{r}}$ :

$$
\frac{\partial P}{\partial f_{r}} \propto \frac{\partial}{\partial f_{r}} \int_{-\infty}^{\infty} W_{t}(t) \cdot d t=0
$$




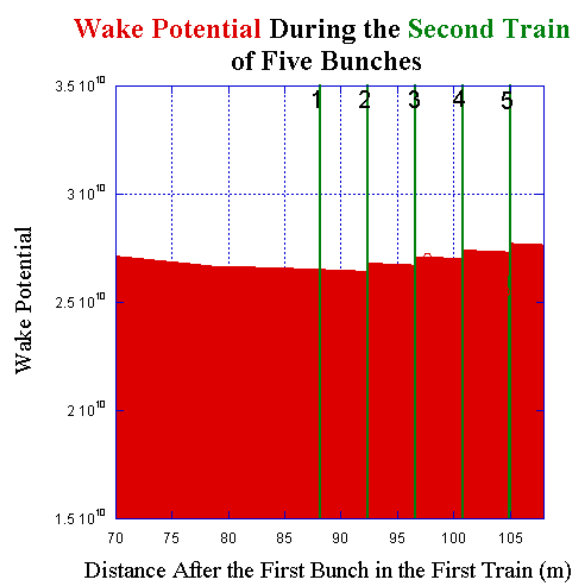

Figure 2: Wake Potential induced during the second trains of five bunches. Note the increase in wake potential with the passage of each bunch in the train.

This condition was achieved in CESR by forcing the sliding joints opening to stay at a fixed position by controlling its water cooling temperature. At this point the resonance frequency of the longitudinal trapped mode of all controlled sliding joints is the same.

\section{THE CONDITIONS FOR MINIMUM BUNCH LENGTHENING}

On the other hand, the length, $\sigma_{l}$, of each bunch depends on the slope of the wake in the sliding joint at the center of the bunch [2].

$$
\sigma_{l} \propto \frac{\partial}{\partial t} W_{t}(t) \mid t \text { at center bunches }
$$

And thus the optimum resonance frequency, $f_{r}$, which will provide minimum bunch lengthening is obtained from:

$$
\frac{\partial}{\partial f_{r}}\left(\frac{\partial}{\partial t} W_{t}(t)\right) \mid t \text { at center bunches }=0
$$

This value of $f_{r}$ is different from the value optimized for minimum heating.

\section{CALCULATION OF THE BUNCH LENGTHENING}

Each bunch circulating the storage ring goes through a hundred sliding joints. At each sliding joint, $s j$, and revolution, $r$, the bunch length, $\sigma l_{r, s j}$, changes by the slope of the wake in the sliding joint according to [2]:

$$
\sigma l_{r, s j}=\sigma l_{r,(s j-1)}\left(1-\frac{\dot{W}_{t}(t)_{s j} \cdot I_{b} \cdot t_{t u r n}}{2 \dot{V}_{r f}}\right)
$$

Where $\dot{W}_{t}(t)_{s j}$ is the time derivative of the wake $W_{t}$ in Equation 2 at sliding joint $s j, I_{b}$ is the current per bunch,
$\dot{V}_{r f}$ is the slope of the $R F$ voltage and $t_{t u r n}$ is the revolution time. For each turn $r$ the index $s j$ goes from 1 to 100 , where $\sigma_{0,0}$ is the equilibrium bunch length.

The wake $\dot{W}_{t}(t)_{s j}$ is calculated self consistently. Its amplitude is updated by the multiplying factor, $F w$, each time the bunch length changes while going through a sliding joint. The factor, $F w$, is the ratio of the bunch Gaussian spectrum to the spectrum of $1 \mathrm{~cm}$ bunch at the sliding joint resonance frequency (see Figure 3). An example of the dependence of $F w$ on the bunch length for $f r=3.575 \mathrm{GHz}$ is seen in Figure 4. Note, the longer the bunch the weaker the excitation of the wake causing to a lower value $F w$.

Using Equations 3 and 5 the optimized resonance frequencies , $f r$ 's, were calculated for each passing bunch. We now find the average of the optimized frequencies for minimum heating of all the bunches, $f r_{a v}$ (min. power), and the average frequency for minimum bunch lengthening, $f r_{a v}(\min . b l)$. We then recalculate the bunch lengthening at these average frequencies.

Figure 5 shows the spread in the bunch lengths when the sliding joint openings were set at $f r_{a v}(\min$. power) for minimum heating. Figure 6 shows a smaller spread at $f r_{a v}(\min . b l)$ that can be achieved by optimizing the opening such that the slope of the wake at the center of the bunch will be minimum.

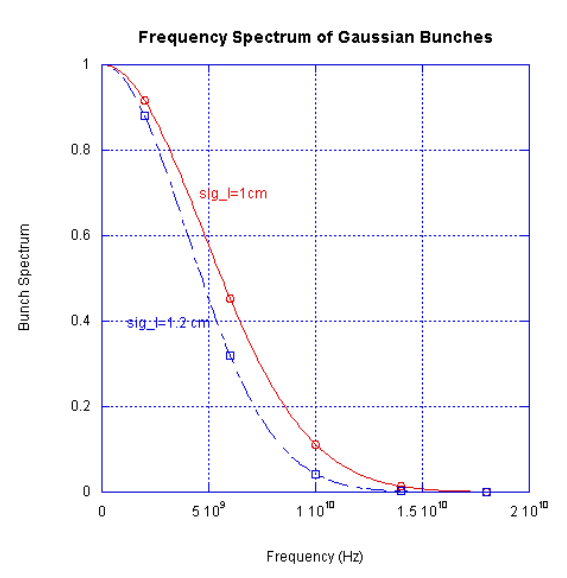

Figure 3: Frequency spectrum of Gaussian bunches. The longer the bunch length the weaker the excitation of the wake potential at each frequency.

\section{CONCLUSIONS}

For nine trains of five bunches at $9 \mathrm{ma} /$ bunch with equilibrium bunch length of $1 \mathrm{~cm}$, the simulation predicts 2.7 times larger difference in bunch lengthening if the sliding joint openings were adjusted to the average resonance frequency optimized for minimum heating versus sliding joint opening adjusted for minimum bunch lengthening. In practice the sliding joints are not identical and the differences are expected to be smaller. 


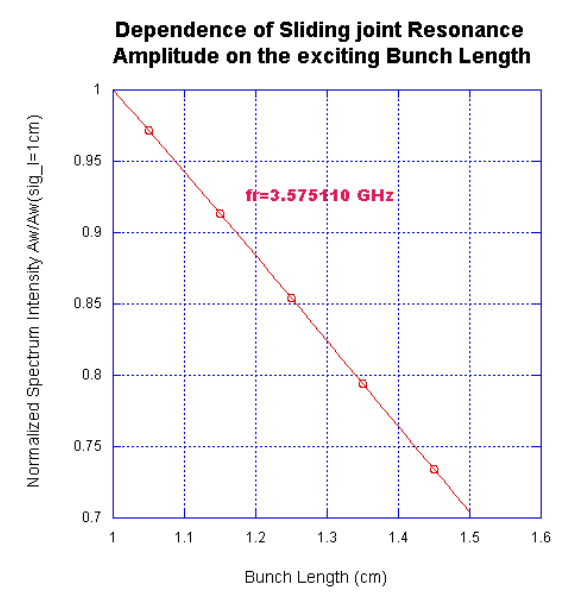

Figure 4: Dependence of the wake potential amplitude, $A w$, on bunch length

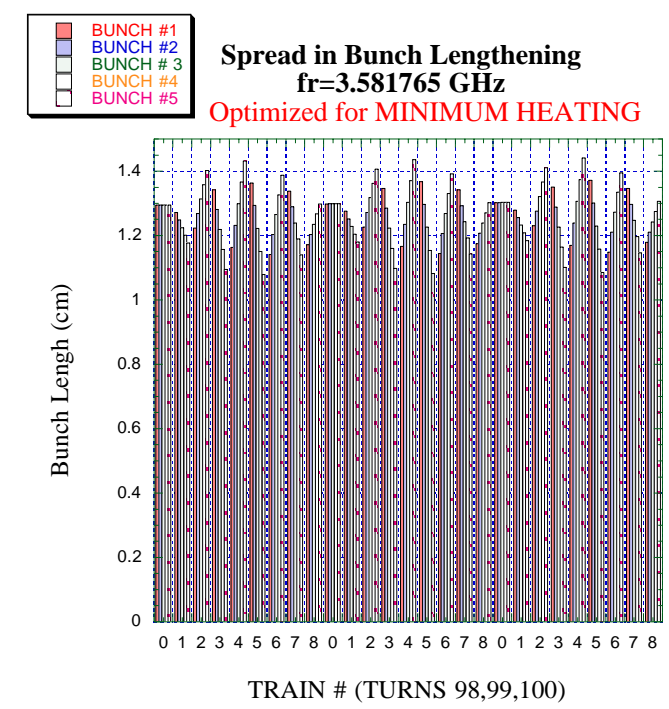

Figure 5: Spread in Bunch Lengthening if all Sliding Joints opening were adjusted to the average resonance for Minimum Heating

The corresponding variations in synchrotron frequency are $21 \%$ for "minimum power" setting and $9.1 \%$ for "minimum bunch lengthening" setting.

The power lost by a single bunch in the "minimum bunch lengthening setting" calculated from the loss parameter, assuming that $R / Q$ does not change, is $30 \%$ larger than that for the "minimum power setting.

The difference in the opening between the two setting is approximately $1.3 \mathrm{~mm}$. Typical difference in sliding joint opening from minimum to maximum power dissipation is $2.5 \mathrm{~mm}$.

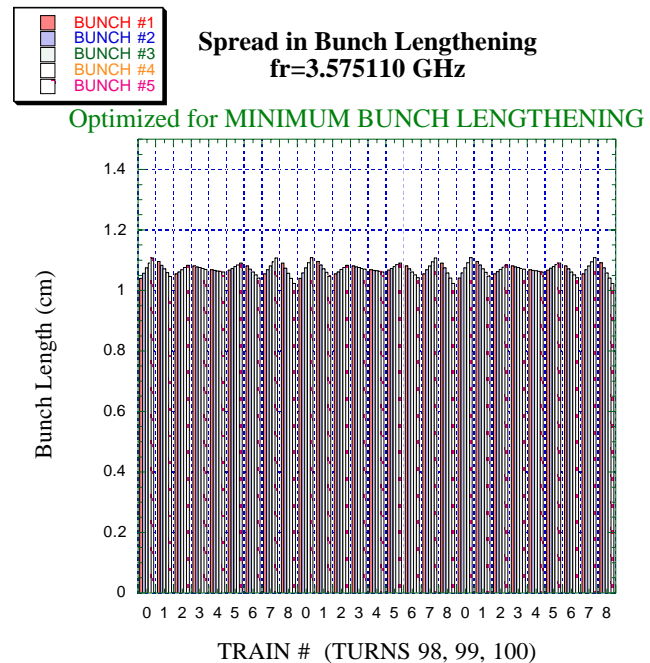

Figure 6: Smaller Spread in Bunch Lengthening if all Sliding Joints were adjusted to the average resonance for minimum Bunch Lengthening

\section{REFERENCES}

[1] Y. Li et al, "Coherent High-Order Heating in CESR Sliding Joints from Multi-Bunched Stored Electron Positron Beams ", This conference

[2] Z. Greenwald, D. L. Rubin, "More about Sliding Joints", CBN 00-14

[3] Z. Greenwald, D. L. Rubin, M. G. Billing, S. B. Peck "Bunch Lengthening for CESR III", e+e- FACTORIES KEK'99

[4] R. Holtzapple, “”, CBN

[5] Z. Greenwald, D. L. Rubin, "Variation in Lengthening of Bunches in Bunch Train Operation in CESR", CBN 00-15 